\title{
Evaluation of Sampling and Testing Efficiencies of Plum pox virus Eradication Programs in Pennsylvania and Ontario
}

A. V. Gougherty, Department of Plant Pathology and Microbiology, K. T. Pazdernik and M. S. Kaiser, Department of Statistics, and F. W. Nutter, Jr., Department of Plant Pathology and Microbiology, Iowa State University, Ames 50011

\begin{abstract}
Gougherty, A. V., Pazdernik, K. T., Kaiser, M. S., and Nutter, F. W., Jr. 2015. Evaluation of sampling and testing efficiencies of the Plum pox virus eradication programs in Pennsylvania and Ontario. Plant Dis. 99:1247-1253.

Plum pox virus (PPV) was first detected in the United States in Pennsylvania in 1999 and in Ontario, Canada in 2000. Following a 10-year survey and eradication program, PPV was officially declared eradicated in Pennsylvania in 2009. Although incidence of PPV was greatly reduced from 2000 to 2008, PPV remains present in Ontario. The objective of this study was to compare how the Pennsylvania and Ontario PPV eradication programs affected the probability of detecting PPV at the leaf, scaffold, tree, and Prunus orchard block scales. A simulation model was developed to evaluate the sampling and testing efficiency of the two programs. At the tree scale, the Pennsylvania sampling and detection protocols had a detection efficiency of $71.8 \%$ compared with $40.5 \%$ for the Ontario program. Several components in the Pennsylvania and Ontario PPV eradication programs affected PPV detection efficiency. A stratified (by tree scaffold) random sampling design did not improve PPV detection

efficiency in either program, compared with a simple random sampling design to select leaves for enzyme-linked immunosorbent assay (ELISA) testing. Detection efficiency for both programs increased with sample size but gains in detection efficiency diminished as sample size increased. There was good agreement (between the commercial ELISA kit used in Pennsylvania and the kit used in Ontario) at the leaf and scaffold scales but not the tree scale. Based on simulation modeling, the Pennsylvania PPV eradication program required that $>2$ PPV-positive trees must be present within a Prunus block to achieve a 95\% probability of correctly detecting PPV at the block scale, whereas the Ontario program required $>5$ PPV-positive trees within a block to achieve $95 \%$ probability of detection. The results from this study have important implications with regard to the efficiency of the two eradication programs to detect PPVpositive trees.
\end{abstract}

Plum pox virus (PPV) is one of the most damaging plant viruses affecting Prunus spp. worldwide (Cambra et al. 2006). It was first detected in North America in 1999 in a peach orchard in Pennsylvania, United States, and in Ontario, Canada in 2000 (Levy et al. 2000; Thompson et al. 2001). PPV is classified as a regulatory pathogen by several phytosanitary organizations, including the North American Plant Protection Organization (NAPPO) (CFIA 2011; NAPPO 2004; USDA 2007). In response to these outbreaks, the U.S. and Canadian governments implemented eradication programs in an effort to minimize the damage to their respective Prunus industries (Thompson 2006; Thompson et al. 2001). In 2009, PPV was officially declared eradicated in Pennsylvania, per NAPPO guidelines (NAPPO 2004; Redding 2009). However, PPV is still present in Ontario and, since 2006, has also been detected in New York State (NAPPO 2013; Snover-Clift et al. 2007). Eradication efforts in New York are ongoing, and no new PPV detections have occurred in New York in the past 3 years. The last remaining regulated areas in New York are located in Niagara County, near the infested area in Ontario, Canada.

The eradication programs in Pennsylvania and Ontario differ in regard to (i) sample size (number of leaves sampled per tree), (ii) enzyme-linked immunosorbent assay (ELISA) kit used to test leaf samples for PPV, (iii) number of PPV-positive leaves required to obtain a positive test result, and (iv) detection thresholds used to determine which Prunus trees or blocks would be eradicated following detection of a PPV-positive Prunus tree.

The Pennsylvania eradication program collected eight leaves per tree, utilizing a stratified sampling design in which two leaves were collected per scaffold. The eight leaves were tested as a bulk sample,

Corresponding author: F. W. Nutter, Jr. (E-mail: fwn@iastate.edu)

Accepted for publication 16 February 2015.

http://dx.doi.org/10.1094/PDIS-07-14-0694-RE

(C) 2015 The American Phytopathological Society with the assumption that one of the eight leaves was required to be positive in order for the bulk sample to be detected as positive. From 1999 to 2006, a Durviz (Valencia, Spain) ELISA test was used to test the bulk leaf samples; however, in 2006, the Pennsylvania PPV eradication program switched to an Agdia (Elkhart, IN) test kit. The Agdia kit utilizes a mixture of several polyclonal antibodies made from recombinant PPV coat proteins (Ravelonandro 2008; Vrient 2006), whereas the Durviz kit utilizes a mix of polyclonal and monoclonal antibodies raised against PPV isolates from Spain. Furthermore, the Agdia kit uses a double-antibody sandwich ELISA procedure (Vrient 2006) whereas the Durviz kit utilizes a doubleantibody sandwich indirect assay (Cambra et al. 1994). The change to the Agdia PPV test kit was prompted by a report that the Agdia kit had fewer false negatives compared with the Durviz kit (Vrient 2006). Upon detecting a PPV-positive tree in Pennsylvania, the eradication program required all Prunus trees within $500 \mathrm{~m}$ of the positive tree to be removed, regardless of health status.

In Ontario, the number of leaves collected per sample increased from 12 to 20 leaves over the length of the eradication program. Leaf collection was stratified equally among tree scaffolds. Bulk leaf samples were tested utilizing a Durviz ELISA test kit. Upon detection of a PPV-positive tree, the positive tree was removed and, depending on the block PPV incidence, the entire block might be removed (Thompson 2006). The threshold for block removal decreased over time, eventually reaching a low of $0.5 \%$ in 2009. An entire block might also be removed, regardless of PPV incidence, if PPV-positive trees were detected in three consecutive years.

European eradication programs. Eradication programs in Europe have also been established, with varying degrees of success (Mumford 2006; Myrta et al. 2006; Ramel et al. 2006; Speich 2006; Zandarski and Zych 2005). In southeast Italy, for instance, after a 15-year PPV eradication campaign, PPV was regarded as essentially eradicated (Myrta et al. 2006). Other countries, such as France, have transitioned to management programs after eradication was no longer deemed feasible (Speich 2006). Though the reasons for failed eradication efforts are varied, in at least one instance, the lack of a reliable laboratory test for PPV was identified as the principle 
reason successful eradication could not be achieved during the initial stages of the epidemic (Mumford 2006). The PPV epidemics in Pennsylvania and Ontario differ from those in Europe in size and magnitude and, consequently, differ in their approach to eradication. The chances of successful eradication of PPV in North America benefit from availability of relatively inexpensive, reliable laboratory tests for PPV (e.g., ELISA and polymerase chain reaction [PCR]) during the onset of the epidemics rather than a reliance on visual surveys, as was used during the initial stages of several European eradication programs (Capote et al. 2009; Mumford 2006).

To date, the sampling and testing protocols used to detect PPV at the leaf, scaffold, tree, and block scales have not been evaluated. Rather than determining the sensitivity of the laboratory tests used in the Pennsylvanian and Ontario eradication programs, the goal here was to develop a method to evaluate different components of a survey program when few data are available. Therefore, the specific objectives of this study were to (i) quantify and compare the sampling and detection efficiencies of the Pennsylvania and Ontario PPV eradication programs; (ii) quantify the level of agreement at the leaf, scaffold, and tree scales for the two PPV eradication programs; (iii) determine the effect of ELISA test kit, number of leaves sampled per tree, and sampling design on PPV detection efficiency; and (iv) determine the number of PPV-positive trees that must be present in a Prunus block for each program to achieve 95 and $99 \%$ probability of detecting PPV at the block scale.

\section{Materials and Methods}

Data collection. Samples of 100 leaves were collected from 19 known PPV-positive peach (Prunus persica) trees in the Niagara peninsula in Ontario, Canada. The 19 trees were initially identified as PPV-positive as part of the Canadian PPV Survey and Eradication Program that was conducted annually under the auspices of the $\mathrm{Ca}-$ nadian Food Inspection Agency (CFIA). In the Canadian program, Prunus trees that initially test positive for PPV by ELISA are retested by PCR to confirm that an individual tree is PPV positive. The 19 confirmed PPV-positive trees for this study were arbitrarily selected from PPV-positive peach trees detected during 2009 (2 trees) and 2010 (17 trees). In collaboration with CFIA survey teams, 100 additional leaves were sampled from each tree within a week after testing positive and prior to removal during the same year. Twenty-five leaves were sampled arbitrarily from each of four scaffolds per tree. Leaves were collected from the second-year growth based on reports that these leaves have the highest virus titer (Stobbs 2009; Thompson 2003). Leaf samples were shipped overnight in accordance with United States Department of Agriculture Animal and Plant Health Inspection Service permit requirements to Iowa State University.

ELISA testing. Each leaf was cut into two equal halves along the midrib: one leaf-half was tested using an Agdia kit (Agdia) and the other was tested using a kit from Durviz. The Agdia kit had been the standard used for detection work in Pennsylvania since 2006 and the Durviz kit is currently used by the Ontario PPV program. Both assays detect the D strain of PPV, which is the predominant strain in Canada and was the predominant strain in Pennsylvania (Thompson 2006; USDA 2007; Wang et al. 2006).

Sap extraction. For the Pennsylvania test protocol, approximately $10 \times(\mathrm{vol} / \mathrm{wt})$ general extraction buffer 4 was added to a half-leaf in each plastic extraction bag (USDA 2013). Half-leaf samples, including one-half of each midrib, were then ground in buffer using a pestle and sap samples were transferred to 1.5-ml Eppendorf tubes that were stored at $-20^{\circ} \mathrm{C}$ until testing (Capote et al. 2009). For the Ontario PPV test protocol, approximately $10 \times(\mathrm{vol} / \mathrm{wt})$ of extraction buffer (phosphate-buffered saline [PBS] $+2 \%$ polyvinyl pyrrolidone- $10+$ $0.2 \%$ skim milk powder) was added to a half-leaf in each extraction bag, after which sample processing and storage was the same as stated above.

Pennsylvania test protocol. Ninety-six-well microtiter plates were coated with a 1:200 concentration of capture antibody diluted in carbonate buffer and allowed to incubate overnight at $4{ }^{\circ} \mathrm{C}$. Plates were then washed with PBS-Tween 20 (PBST) with a microplate washer (Model ELx405; Biotec Inc., Winooski, VT), and wells were loaded with 100- $\mu$ l aliquots of leaf sample. Each plate had two positive control test wells, two negative control test wells, and two buffer control wells. Plates were incubated overnight at $4^{\circ} \mathrm{C}$, then again washed with PBST, after which wells were loaded with $100 \mu 1$ of 1:200 concentration enzyme conjugate diluted in RUB3 and allowed to incubate for $2 \mathrm{~h}$ at room temperature (approximately $21^{\circ} \mathrm{C}$ ). Plates were then washed a final time and loaded with $100 \mu$ l of p-nitrophenol (PNP) at $1 \mathrm{mg} / \mathrm{ml}$ and incubated in darkness for $1 \mathrm{~h}$ before absorbance was read at $405 \mathrm{~nm}$ with a plate reader (Elx800; Biotec Inc.). Samples with readings that were twice the mean of the absorbance of the negative control wells were deemed positive for PPV. Plates that did not have positive results for both PPV control wells were redone.

Ontario test protocol. Plates were coated with 1:100 capture antibody in carbonate buffer and allowed to incubate overnight, then washed with PBST and loaded with $100 \mu \mathrm{l}$ of leaf extract. After a 100- $\mu \mathrm{l}$ aliquot of 1:1000 dilution of monoclonal and polyclonal antibody diluted in PBS plus $0.5 \%$ bovine serum albumin was added to each well, plates were incubated for $2 \mathrm{~h}$, followed by rewashing with PBST and loading wells with a 1:1000 dilution of antimouse immunoglobulin conjugated with alkaline phosphatase diluted in PBS, and incubated for $2 \mathrm{~h}$. Plates were washed a final time with PBST and wells were loaded with PNP solution at $1 \mathrm{mg} / \mathrm{ml}$. After incubating for an hour in darkness, absorbance was read at $405 \mathrm{~nm}$ using a plate reader (Elx800).

Comparing test kits used in the Pennsylvania and Ontario. Strength of agreement between the Agdia (Pennsylvania) and Durviz (Ontario) ELISA kit results at the leaf, scaffold, and tree scales were assessed using McNemar's $\chi^{2}$ test for paired data. McNemar's $\chi^{2}$ statistic $\left(\chi^{2}\right)$ assesses the relationship between two independent tests of paired data in a two-by-two contingency table (McNemar 1947). McNemar's $\chi^{2}$ statistic tests the null hypothesis of whether the outcomes of the two tests are independent. McNemar's test was calculated as $\chi^{2}=(a-b)^{2} / a+b$, where $a$ is the number of samples that tested positive by test 1 and negative by test 2 , and $b$ is the number of samples that tested negative by test 1 and positive by test 2 .

Cohen's Kappa statistic (к) was used to determine how well the test kit results agreed when testing half-leaves from the same sampled leaf. Cohen's $\kappa$ is a measure of concordance (Cohen 1968) and is calculated by comparing the observed and expected frequencies of agreement as $\kappa=p_{o}-p_{e} / 1-p_{e}$, where $p_{o}$ is the observed proportion of agreement and $p_{e}$ is the proportion of agreement expected by chance (Cohen 1968). Results for $\kappa$ range from 0 to 1 , where 0 indicates no correlation and 1 indicates perfect correlation. Cohen's $\kappa$ was calculated using the $\kappa$ option in PROC FREQ in SAS. Scaffolds and trees were considered PPV positive if at least one leaf on a scaffold or one scaffold on a tree tested positive for PPV, respectively.

Comparison of sampling and detection efficiencies. To determine the effect of key sampling and testing components of both programs on PPV detection efficiency, a nonparametric Monte Carlo simulation model was developed. Approximations of detection efficiency were computed by sampling from the ELISA test results obtained from both ELISA kits for the same leaves, scaffolds, and trees. Of the 19 trees that were intensively resampled, no PPVpositive leaves were detected by either of the two test kits for 2 of the 19 trees. Two other trees in the sample tested positive using the Agdia kit but not the Durviz kit, and two other trees tested positive for PPV using the Durviz kit but not the Agdia kit. Hence, 13 of 19 trees had PPV-positive leaves that were detected by both the Pennsylvania and Ontario ELISA kit protocols. Therefore, the simulation study was run on the ELISA results from these 13 trees. Factors included in the approximation of detection efficiencies were (i) the ELISA test kit used, (ii) sampling design, (iii) number of leaves sampled per tree, and (iv) number of PPV-positive leaves required for a bulk sample to test positive for PPV. Cross classifications of these factors resulted in a total of 200 individual protocols for disease detection. The objective of the Monte Carlo simulation study was to determine the probability of detection under each of these protocols.

The simulation study was conducted as follows. A random leaf sample was collected (without replacement) from each of the 13 trees 
previously classified as PPV positive (by PCR) and for every combination of the first three factors (excluding the number of PPVpositive leaves required for a positive result). Two sampling designs were evaluated: (i) a simple random sampling design and (ii) a stratified random sampling design. For simple random sampling, leaves were selected at random among all 100 leaves sampled and individually tested for PPV per tree from all four scaffolds. For stratified random sampling, an equal number of leaves (USDA 2007) was randomly sampled from each of the four scaffolds. Sample sizes varied between 4 and 40 leaves per tree (in 4-leaf intervals; i.e., 4, 8, 12, 16, and so on). Increments of four leaves were selected to create a balanced sampling design within each scaffold when a stratified random sampling design was employed and to allow for direct comparison between the two sampling designs. The number of PPV-positive leaves on a given tree assumed to be necessary in order for a bulk sample to test positive for PPV was varied from one to five.

We desired 95\% Monte Carlo interval approximations to have half-widths of 0.00033 or less. This means, for example, given an approximated probability of 0.06371 , we are $95 \%$ confident that true probability of detection is contained in the interval $(0.06338$, 0.06404). A Monte Carlo sample size of 500,000 was found to be efficient to produce this level of precision for every combination of factors in this simulation study. Detection efficiency was calculated as follows: detection efficiency $=$ (total number of iterations resulting in PPV-positive trees/total number of iterations) $\times 100$.

The probability of correctly detecting a PPV-positive block was calculated by simulating various numbers of PPV-positive trees within a Prunus block. To do so, we used the principles of inclusion and exclusion to determine the probability of detecting at least one PPV-positive tree when two or more PPV-positive trees are present within a block (Roberts and Tesman 2011). The probability of correctly detecting that a block was PPV-positive when one, two, or three PPV-positive trees are present was calculated using set theory, specifically:

$$
\begin{aligned}
& \mathrm{P}(A)=\mathrm{P}(A) \\
& \mathrm{P}(A \cup B)=\mathrm{P}(A)+\mathrm{P}(B)-\mathrm{P}(A \cup B) \\
& \begin{aligned}
\mathrm{P}(A \cup B \cup C)= & \mathrm{P}(A)+\mathrm{P}(B)+\mathrm{P}(C)-\mathrm{P}(A \cup B)-\mathrm{P}(A \cup C)-\mathrm{P}(B \cup C) \\
& +\mathrm{P}(A \cup B \cup C)
\end{aligned}
\end{aligned}
$$

where $\mathrm{A}, \mathrm{B}$, and $\mathrm{C}$ represent one, two, and three trees, respectively; $\mathrm{P}(A)$ is the probability of detecting a block as PPV positive when one PPV-positive tree is present within a Prunus block; $\mathrm{P}(A \cup B)$ is the probability of detection when two PPV-positive trees are present within a block; and $\mathrm{P}(A \cup B \cup C)$ is the probability of detection when three PPV-positive trees are present within a block, and so on. Probabilities were calculated for up to $10 \mathrm{PPV}$-positive trees per block.

\section{Results}

Comparing overall detection efficiency. Based on simulation results, the Pennsylvania program had a higher overall PPV detection efficiency at the tree scale $(71.7 \%)$ than the Ontario program $(40.5 \%)$ (Fig. 1A and B). This indicates that, when the same leaf samples were split in half and each half was tested separately, the Pennsylvania sampling and detection program would have a detection probability of $71.7 \%$ at the tree scale (for known PPV-positive trees previously confirmed by PCR), whereas the Ontario sampling and testing program would have correctly detected approximately $40.5 \%$ of the population of known PPV-positive trees tested.

ELISA test kit comparison. The Agdia ELISA kit detected more than twice as many PPV-positive leaf samples among all 19 intensively sampled trees as the Durviz kit. In total, the Agdia kit detected 244 PPV-positive leaves (13.2\% of all leaves), whereas the Durviz kit detected 115 PPV-positive leaves (6.2\%) from the same trees, using the same split-leaf subsamples (Table 1). Of the 72 scaffolds tested, using the Agdia kit, 44 of the scaffolds $(61.1 \%)$ tested positive for PPV whereas $23(31.9 \%)$ tested positive for PPV using the Durviz kit (Table 1). Of the 19 PPV-positive trees intensively sampled in our study, 15 were detected as positive by either the Agdia or Durviz kits (Table 1). Thirteen trees were detected as PPV positive by both test kits.
Using the Pennsylvania protocol, the incidence of PPV-positive leaves within scaffolds averaged $14.1 \%$, whereas incidence of PPV-positive leaves within scaffolds using the Ontario protocol averaged $6.5 \%$ (Table 2). The lowest incidence within scaffolds of known PPV-positive trees was $0 \%$ for both protocols, whereas the highest incidence was 64.0 and $32.0 \%$ for Pennsylvania and Ontario protocols, respectively (Table 2). The large standard deviations for both the Pennsylvania (18.1\%) and the Ontario $(9.8 \%)$ PPV sampling and testing programs demonstrate the degree of heterogeneity in the incidence of PPV-positive leaves within scaffolds.

Average incidence of PPV-positive leaves within Prunus trees ranged from 0 to $44.0 \%$ using the Agdia test kit, with an average of $13.3 \%$, whereas incidence using the Durviz test kit ranged from 0 to $35.7 \%$, with an average of $6.9 \%$. Standard deviations for incidence at the tree scale were also high (13.8 and 9.3\% for the Agdia and Durviz kits, respectively).

Agreement between ELISA results using the Agdia and Durviz kits was statistically significant at the leaf and scaffold scales $(P<0.0001$ and $P=0.019$, respectively), based upon results of McNemar's $\chi^{2}$ test for paired data (Table 3 ). The $\chi^{2}$ test at the tree scale, however, was not significant $(P=1.0)$, indicating a nonsignificant relationship between the two tests at the tree scale. The large $P$ value here
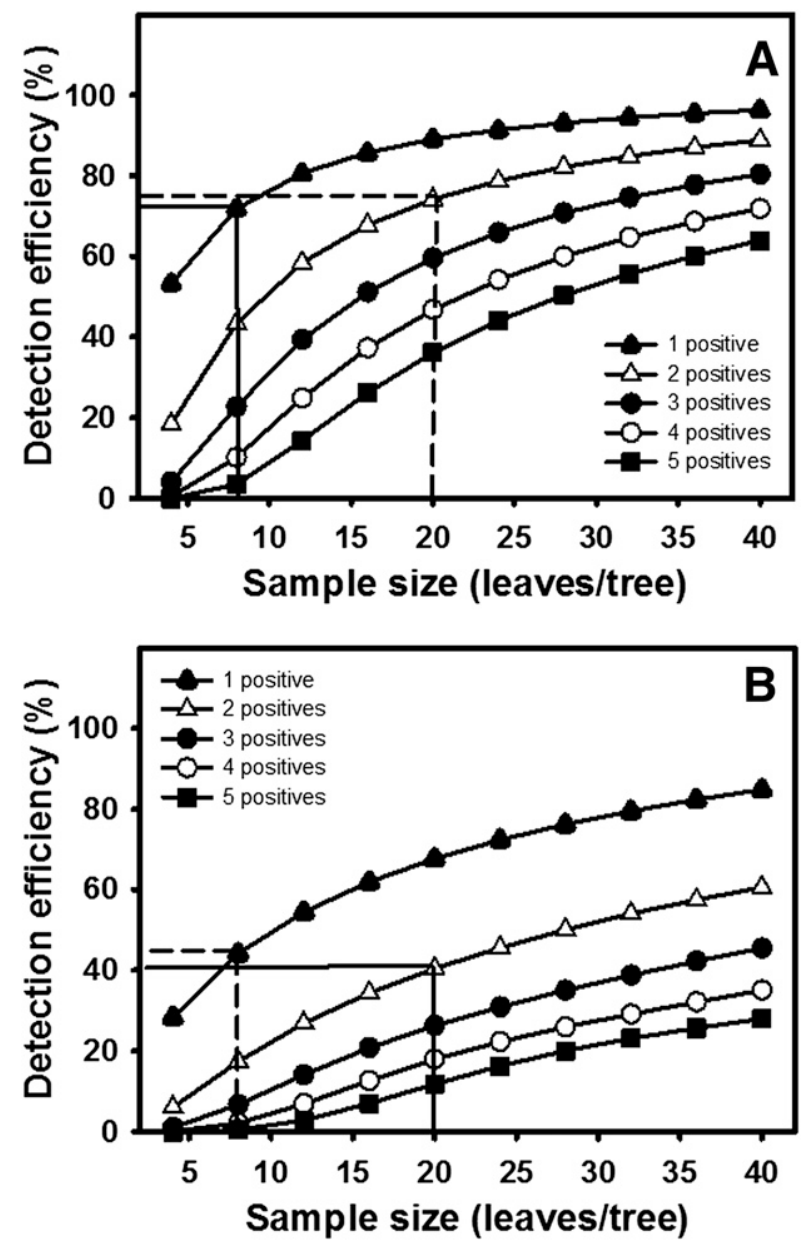

Fig. 1. Detection efficiency (\%) of $\mathbf{A}$, the Pennsylvania Plum pox virus (PPV) sampling and testing program and $\mathbf{B}$, the Ontario PPV sampling and testing program based on 500,000 iterations of a model simulating the resampling (without replacement) of 13 known PPV-positive trees. Solid reference lines depict the actual program detection efficiency (i.e., Pennsylvania in A and Ontario in B) and dashed reference lines represent the hypothetical detection efficiency of the other program (i.e., Ontario in $A$ and Pennsylvania in B). The Pennsylvania sampling and testing system consisted of an 8-leaf bulk sample requiring at least 1 PPV-positive leaf in a bulk sample to test positive for PPV, and the Ontario sampling and testing system consisted of a 20-leaf bulk sample requiring 2 PPV-positive leaves in a bulk sample to test positive for PPV. Curves show simulated detection efficiency given $n=1$ to 5 PPV-positive leaves per bulk sample. 
is likely the result of a relatively small sample size (19 trees). Cohen's $\kappa$ was highest at the scaffold scale $(\kappa=0.40)$, followed by the leaf $(\kappa=0.38)$ and tree $(\kappa=0.37)$ scales (Table 3$)$. Thus, the correlation between results from Pennsylvania and Ontario PPV sampling and testing systems was highest at the scaffold scale, followed by the leaf and tree scales.

Results from simulation modeling revealed that the most important factor affecting PPV detection efficiency was the ELISA kit used to test Prunus leaves. The Pennsylvania PPV protocol, as mentioned above, had an overall detection efficiency of $71.7 \%$ at the tree scale. Had the Pennsylvania program kept all factors the same except for the ELISA kit used (i.e., eight-leaf bulk sample requiring one PPVpositive leaf, with a stratified sampling design) but instead used the Durviz ELISA kit, detection efficiency at the tree scale would have dropped to $44.2 \%$, which is significantly lower (Fig. 1A and B).

Table 1. Comparison of the Pennsylvania and Ontario Plum pox virus (PPV) eradication sampling and testing protocols on PPV incidence at the leaf, scaffold, and tree scales in peach (Prunus persica) when the same leaf samples were tested using each region's PPV sampling and testing protocols

\begin{tabular}{lcc}
\hline Scale $^{\mathbf{a}}$ & Pennsylvania & Ontario \\
\hline Leaf & & \\
Number PPV positive & 244 & 115 \\
Total $(n)$ & 1,846 & 1,846 \\
PPV positive (\%) & 13.2 & 6.2 \\
Scaffold & 44 & 23 \\
$\quad$ Number PPV positive & 72 & 72 \\
Total $(n)$ & 61.1 & 31.9 \\
PPV positive (\%) & & \\
Tree & 15 & 15 \\
Number PPV positive & 19 & 19 \\
Total $(n)$ & 78.9 & 78.9 \\
PPV positive $(\%)$ &
\end{tabular}

a Nineteen Prunus trees were intensively sampled (100 leaves per tree) and each leaf was split into two halves at the midrib, with each half-leaf being tested using either the Pennsylvania or the Ontario sampling and testing protocol.

Table 2. Comparison of the Pennsylvania and Ontario Plum pox virus (PPV) eradication, sampling, and testing protocols on PPV leaf incidence at the scaffold and tree scales in peach (Prunus persica) when the same leaf samples were tested using both regions' protocols

\begin{tabular}{lcc}
\hline Scale $^{\mathbf{a}}$ & Pennsylvania (\%) & Ontario (\%) \\
\hline Scaffold & & \\
Range & $0-64.0$ & $0-32.1$ \\
Mean & 14.1 & 6.5 \\
$\quad$ Standard deviation & 18.1 & 9.8 \\
Tree & & \\
Range & $0-44.0$ & $0-35.7$ \\
Mean & 13.3 & 6.9 \\
Standard deviation & 13.8 & 9.3 \\
\hline
\end{tabular}

a Nineteen Prunus trees were intensively sampled (100 leaves per tree) and each leaf was split down the midrib into two halves, with each half-leaf being tested using either the Pennsylvania or the Ontario sampling and testing protocol.

Table 3. Agreement of Agdia (Pennsylvania) and Durviz (Ontario) enzymelinked immunosorbent assay (ELISA) kits at the leaf, scaffold, and tree scales on the detection of Plum pox virus (PPV) of peach (Prunus persica) using McNemar's $\chi^{2}$ test $\left(\chi^{2}\right)$ and Cohen's $\kappa^{\mathrm{a}}$

\begin{tabular}{lcccc}
\hline Scale & Number & $\boldsymbol{\chi}^{\mathbf{2}}$ & $\boldsymbol{P}$ value & $\boldsymbol{\kappa}$ \\
\hline Leaf & 1,846 & 80.71 & $<0.0001$ & 0.38 \\
Scaffold & 72 & 5.50 & 0.02 & 0.40 \\
Tree & 19 & 0.00 & 1.00 & 0.37 \\
\hline
\end{tabular}

a Independence between the two ELISA test kits was rejected when $P<0.05$ for the $\chi^{2}$ value; $\kappa$ is a measure of correlation, which can range from 0 to 1 , where 1 is perfect agreement.
Likewise, by leaving all other factors in the Ontario program the same but substituting the Agdia ELISA kit for the Durviz kit, the Ontario sampling program (i.e., 20-leaf bulk sample requiring a minimum of 2 PPV-positive leaves and employing a stratified random sampling design) would have resulted in a detection efficiency of $74.1 \%$ at the tree scale, which is significantly higher than the level of detection efficiency achieved using the Durviz kit (40.5\%).

Sample design. No appreciable effect was detected whether using a random sampling design or a stratified (by scaffold) random sampling design. Utilizing the Ontario sampling protocol, detection efficiency using a random sampling design was $40.6 \%$, compared with $40.5 \%$ for the stratified random sampling design (Fig. 2). Similarly, the Pennsylvania sampling protocol with a random sampling design was $70.4 \%$, compared with $71.8 \%$ using a stratified sampling design.

Sample size. For both sampling and testing systems, detection efficiency increased as sample size increased (Fig. 3A), although gains in detection efficiency diminished as sample size increased (Fig. 4). These findings were consistent for both the Pennsylvania and Ontario sampling and testing programs using either sampling design.

When the number of PPV-positive leaves that would be needed for a bulk leaf sample to test positive for PPV was increased in proportion to the sample size, detection efficiency decreased slightly as sample size increased (Fig. 3B). For instance, if detection sensitivity for the Pennsylvania program was assumed to be $1 \mathrm{PPV}$-infected leaf per 10-leaf sample, detection efficiency at the tree scale would increase to $77 \%$ (Fig. 3B). However, when the same ratio was maintained, detection efficiency for 20,30 , or 40 bulk leaf samples decreased to 74,73 , and $72 \%$, respectively.

Number of PPV-positive leaves required for a bulk leaf sample to test positive for PPV. Detection efficiency was highest in simulations for bulk leaf samples that required only a single PPV-positive leaf within a bulk sample (Fig. 3A). Detection efficiency decreased with each additional PPV-positive leaf that was assumed to be required for a bulk sample to test positive. The discrepancy in detection efficiency between requiring one and two PPV-positive leaves was greater than that between requiring four and five PPV-positive leaves. This was true for simulations using either of the two PPV sampling and testing systems.

Detection efficiency at the block scale. If a Prunus block contained only a single PPV-positive tree, the Pennsylvania sampling and testing system would have a $71.7 \%$ probability of correctly determining that the block was PPV positive, thereby triggering eradication of that block as well as all other Prunus trees within $500 \mathrm{~m}$. The Ontario testing and sampling program, however, had a $40.5 \%$ probability of

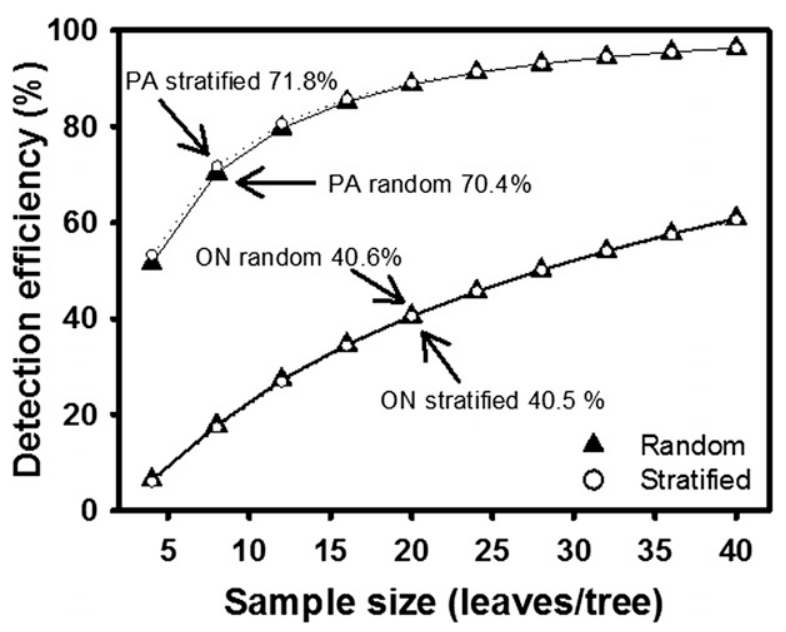

Fig. 2. Effect of sample design on Plum pox virus (PPV) detection efficiency (\%) for the Pennsylvania (PA) and Ontario (ON) PPV sampling and testing programs as estimated by 500,000 iterations of a model simulating the resampling (without replacement) of 13 known PPV-positive trees. Triangles represent a random sample design and circles represent a stratified (by scaffold) sample design. 
detecting a PPV-infected block as positive when only one PPVpositive tree was present. Therefore, the Ontario program would correctly trigger the implementation of their PPV eradication policy $40.5 \%$ of the time. If two PPV-positive trees were present within a Prunus block, detection efficiency at the block scale for the Pennsylvania program was $91.9 \%$, compared with $64.6 \%$ for the Ontario program (Fig. 5). To achieve 95 or $99 \%$ probability of detecting PPV, a minimum of three or four PPV-positive trees, respectively, within a block would need to be present using the Pennsylvania system. In contrast, the Ontario system would require at least six PPV-positive trees within a Prunus block to achieve a $95 \%$ probability or nine PPVpositive trees to achieve a $99 \%$ probability of detecting PPV.

\section{Discussion}

To our knowledge, this is the first study of its kind to evaluate and compare two regional-scale sampling and testing programs charged with eradicating a plant pathogen. By quantifying the sampling and detection efficiencies of the Pennsylvania and Ontario PPV eradication programs, new science-based information was obtained that will be useful for improving current PPV-eradication programs (e.g., New York), as well as helping to ensure a higher probability of successful PPV management in Ontario, Canada.

When a nonindigenous plant pathogen such as PPV is first detected and confirmed in a new location, a critical question immediately arises: can the new threat be successfully eradicated? Eradication
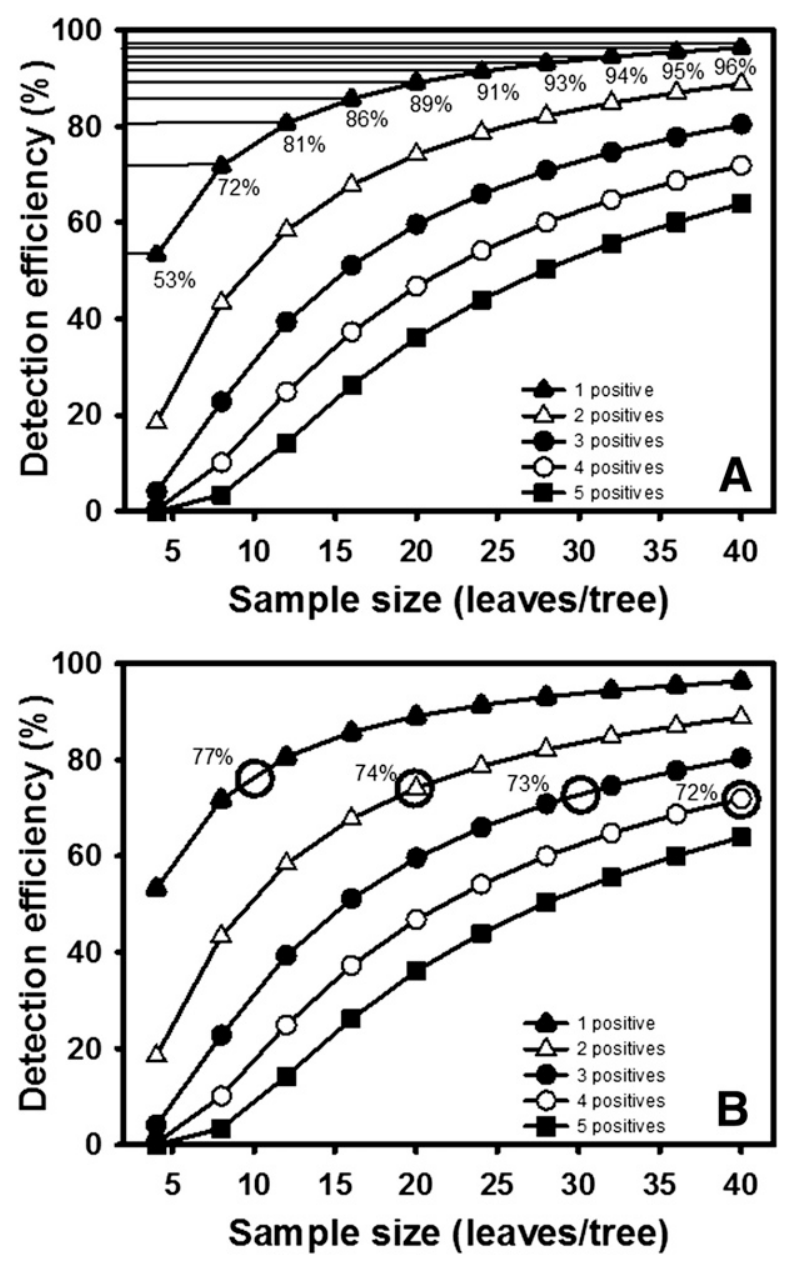

Fig. 3. Effect of sample size on Plum pox virus (PPV) detection efficiency (\%) of the Pennsylvania PPV sampling and testing program using a simulation model $(500,000$ iterations) assuming A, a constant number of PPV-positive leaves in a bulk sample across all sample sizes (i.e., 1) and B, a constant ratio of positive leaves to the number of Prunus leaves in a bulk sample (i.e., 1:10). Detection efficiency increased with increasing sample size if the number of leaves was kept constant (A) but decreased with sample size when the leaf ratio was kept constant (B). programs are often established on the basis of incomplete biological information (Nutter 2007). It is essential that robust sampling and testing protocols are developed to correctly identify which plants or plantings are infected (Zadoks and Schein 1979). Furthermore, an eradication program must be continually reevaluated to ensure program success (Nutter 2007; Nutter et al. 2011). We have demonstrated that simulation modeling can help to evaluate the sampling and testing protocols on which action thresholds are based. Simulation models allow researchers and policy makers to ask "what if" questions concerning the impact of altering sampling and test procedures on optimizing detection efficiency. Although the present study was conducted after PPV eradication programs in Ontario and Pennsylvania were first initiated, future plant disease eradication programs would likely benefit by using similar simulation approaches during the early stages of an epidemic. Such simulation approaches could also be periodically performed after the initiation of a new eradication program to evaluate the performance of the sampling and testing protocols currently in use. This information could then be used to amend sampling and testing protocols as needed to improve detection efficiency.

Probability of PPV detection at the block scale. An important finding of our study was to pinpoint the number of PPV-positive trees that are needed at the block scale for the Pennsylvania or Ontario PPV eradication programs to correctly detect PPV-positive Prunus

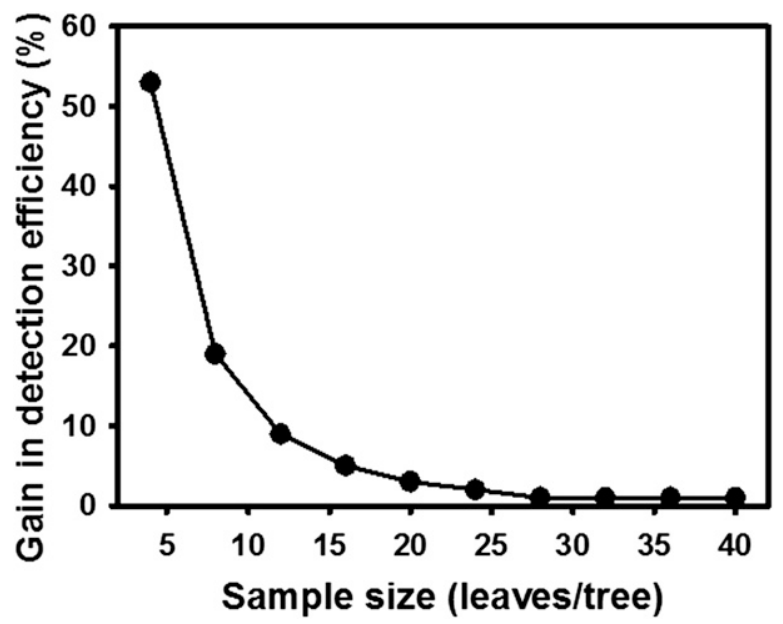

Fig. 4. Use of a simulation model (500,000 iterations) to determine the gain in Plum pox virus (PPV) detection efficiency (\%) utilizing the Agdia kit and requiring only a single PPV-positive leaf for a bulk leaf sample to test positive for PPV. As sample size increases, the gain in PPV detection efficiency decreases.

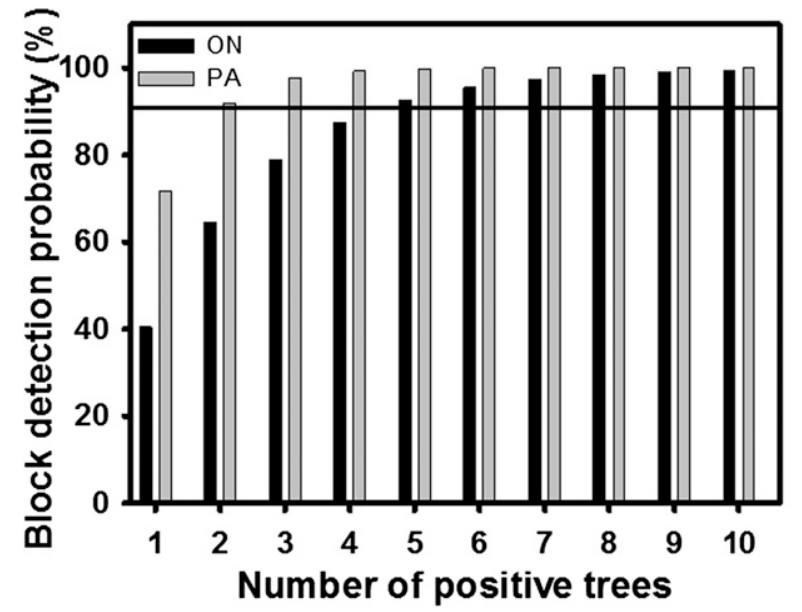

Fig. 5. Effect of increasing the actual number of Plum pox virus (PPV)-positive Prunus trees per block on the probability of detecting PPV at the block scale using the Pennsylvania (PA) and the Ontario (ON) PPV sampling and testing programs. The solid black line represents $90 \%$ detection probability. 
blocks. The block scale is a key management unit for PPV eradication; therefore, the probability of detecting PPV at the block scale affects whether or not eradication action thresholds will be triggered by either program. By requiring more PPV-positive trees per block to achieve detection probabilities of 90,95 , or $99 \%$, the Ontario PPV eradication program would result in a higher number of nondetected (misdiagnosed) trees at the block scale compared with the Pennsylvania eradication program. For example, if one Prunus block in Pennsylvania and another in Ontario both consisted of 200 trees and each contained two confirmed PPV-positive trees (1\% incidence), the Pennsylvania program would have a $91.9 \%$ probability of detecting one or both PPV-positive trees in the block, whereas the Ontario program would have a $64.6 \%$ probability of detecting one or both PPV-positive trees.

Even if PPV is correctly detected, a PPV-positive block detected in Ontario would not necessarily be removed unless PPV incidence in the affected block exceeded $0.5 \%$, or if the block had tested positive for PPV for three consecutive growing seasons. Thus, the Ontario program allows some PPV-positive blocks to remain in production. These two PPV action thresholds for block removal are less stringent than the Pennsylvania zero-tolerance policy. These less stringent policies, however, were needed to maintain viability of the Ontario Prunus industry, which is densely concentrated in the Niagara Peninsula. Furthermore, Ontario faced a greater initial challenge than Pennsylvania, because more than twice as many PPV-positive trees were detected during the first survey year. In contrast, Pennsylvania had fewer PPVpositive trees in the first year of their eradication program, and also had a Prunus industry that was sufficiently dispersed to allow for the eradication of all Prunus trees within $500 \mathrm{~m}$ of a PPV-positive tree (which was not the case in Ontario). Moreover, a higher number of PPV-misdiagnosed trees (at the tree and block scales) would allow some additional PPV-positive trees and blocks in Ontario to remain in production. This is undesirable epidemiologically, because PPVpositive trees that are misdiagnosed as being PPV negative would allow some Prunus trees and blocks to continue to serve as sources of inoculum for PPV acquisition and transmission by aphid vectors (Gougherty and Nutter 2015; Madden et al. 2007).

Agreement between ELISA test kits. Although there was agreement between the two PPV ELISA test kits at the leaf and scaffold scales, the two sampling and testing programs did not agree at the tree scale. This was likely due to the fact that even though these trees were previously confirmed to be PPV positive by both ELISA and PCR tests, four trees had 5 or fewer PPV-positive leaves (out of 100) that tested positive for PPV. Thus, the probability of selecting a single positive leaf in an 8-leaf sample (Pennsylvania) or 2 positive leaves in a 20-leaf sample (Ontario), when the incidence of PPV-infected leaves was $<5 \%$, is low. Still, the lack of agreement between the results of the two programs is disconcerting because tree removal protocols are highly dependent upon the ability of survey programs to accurately detect PPV infection. The factor most responsible for lack of agreement at the tree scale was the ELISA kits used, because the Agdia PPV ELISA kit detected more than twice as many PPVpositive leaf subsamples as the Durviz kit. Although it is possible that the relatively greater success achieved by the U.S. eradication programs in Pennsylvania was due to the higher PPV detection efficiency, it must be kept in mind that our study was conducted in 2009 and 2010 and that both countries had used the Durviz ELISA kit until 2006 Thus, prior to 2006, it is likely that other factors in the Pennsylvania program such as replant restrictions, differences in sampling protocols, and the more stringent postdetection eradication policies contributed to the successful eradication of PPV in Pennsylvania. The two eradication programs also differ in a number of other important regulatory features, including the definition of a "block." In Canada, a block is defined as the planting of a single cultivar of a given Prunus sp., whereas a "block" in the United States is defined as the planting of a given Prunus sp.

The Pennsylvania and Ontario PPV eradication programs. Our findings have important implications with regard to the successful eradication of PPV. The more aggressive eradication removal policy in Pennsylvania (i.e., the removal of all Prunus trees within $500 \mathrm{~m}$ of a PPV-positive block, regardless of PPV status) likely compensated for any failure to detect all PPV-positive trees in a Prunus block, resulting in a lower annual initial inoculum in Pennsylvania.

It is reasonable to assume that, after 2006 (when the United States switched from the Durviz to the Agdia PPV ELISA kit), the Ontario program had a lower detection efficiency than the Pennsylvania program. Examination of the within-block spatial and temporal patterns of PPV-positive trees in Ontario revealed that new PPV-positive trees were often detected within Prunus blocks that were found to be PPV positive in previous years, even after all detected PPV-positive trees had been removed (Gougherty and Nutter 2015). Ordinary runs analysis of PPV-positive trees within Prunus blocks revealed that the PPV-positive trees were significantly clustered in $25 \%$ of Prunus blocks in Ontario that were intensively surveyed and mapped (Gougherty and Nutter 2015). These findings suggest that nondetected (misdiagnosed) PPV-positive trees affected the health status of Prunus blocks for up to 3 years after PPV-positive trees were removed (Gougherty and Nutter 2015). This is troubling for PPV eradication programs based on annual within-block incidence thresholds and supports our contention that some PPV-positive trees remained undetected in Ontario for up to 3 years. Undetected PPV-positive trees will continue to serve as sources of PPV inoculum until they are eventually detected and removed in subsequent survey years.

Sample size. Detection efficiency at the tree scale increased with increasing sample size in both PPV sampling programs as well as for either sampling design (random or stratified random). However, it is important to consider that, as sample size increases, the number of PPV-positive leaves required for a bulk sample to test positive for PPV also increases. Based upon the assumption that 1 PPV-positive leaf is required in an 8-leaf bulk sample in the Pennsylvania program and 2 PPV-positive leaves are required in a 20-leaf bulk for the Ontario program, it can be assumed that approximately 1 additional PPVpositive leaf is required for each 8- to 10-leaf increase in sample size. One published report confirms that at least 2 PPV-positive leaves are required for a 16-leaf bulk sample to test positive for PPV (Hughes et al. 2002). Thus, there may be little benefit in increasing sample size to increase PPV detection efficiency if a ratio of $1: 8$ or $1: 10$ PPVpositive leaves in a bulk sample is maintained. In fact, our simulation study showed that, when increasing sample size using a 1:10 ratio of PPV-infected to healthy leaves, PPV detection efficiency at the tree scale actually decreased. If more sensitive PPV detection tools were employed to detect a single PPV-infected leaf in a 40-leaf bulk sample, then detection efficiency could hypothetically be increased from its present level of 71.8 to $96.3 \%$ in the Pennsylvania program (Fig. 3A). Based on our study, two factors that would most improve PPV detection efficiency in future PPV eradication programs would be (i) choice of commercially available ELISA kit or (ii) adoption of a more sensitive PPV detection test that could more accurately detect one PPV-infected leaf in larger bulk samples.

Sample design. Sampling design (random versus stratified random [by scaffold]) was found to have little effect on PPV detection efficiency. However, the fact that both the Pennsylvania and Ontario sampling and testing systems detected only 4 of 19 trees in which all four scaffolds (within the same tree) tested positive for PPV supports the continuation of using a stratified random sampling design. Also, the $1.43 \%$ gain in PPV detection efficiency using a stratified (by scaffold) random sample design is a significant gain after running 500,000 iterations. A more extensive study is needed to evaluate whether the extra time and resources needed to specifically sample each scaffold of a Prunus tree is worth any gains in PPV detection efficiency.

Based upon our simulation study, it would be best to require only a single PPV-positive leaf per bulk sample to test positive for PPV. One way to optimize virus detection in a bulk leaf sample that contains only a single PPV-positive leaf is to sample Prunus trees when the virus titer is likely to be highest (Stobbs 2009). PPV titer within Prunus trees is known to vary throughout the growing season, often in an inverse relationship to temperature (Stobbs 2009; Thompson 2003; USDA 2007). In a 2001 study, Thompson and associates found that PPV titer in Prunus trees in Ontario was lowest between late July and late September (Thompson 2003). Despite this finding, both the 
Pennsylvania and Ontario PPV survey programs continue to conduct PPV surveys throughout the mid- to late summer months. Because PPV-positive trees were intensively resampled 10 to 14 days after the trees were initially sampled and then retested by ELISA, this may be the reason that both the Pennsylvania and Ontario sampling and testing programs deemed only 13 of the 19 trees intensively resampled positive for PPV. In a related study, Gougherty and Nutter (2015) recently reported the presence of a seasonal periodicity in the percentage of PPV-infected trees that were detected during the growing season in both Pennsylvania and Ontario, as shown by a higher percentage of PPV-positive trees being detected in May and June, when temperatures were relatively cooler $(60.5 \%)$, compared with $38.2 \%$ in July and August, when seasonal temperatures were higher (Gougherty and Nutter 2015).

It should be acknowledged that this study was based upon a rather small number of PPV-infected trees. Still, the number of trees used in our study was sufficient to document and model the heterogeneity in PPV incidence that is present at the leaf, scaffold, and tree scales. The high degree of heterogeneity in the detection of PPV-positive leaves within scaffolds of known PPV-positive trees points to the importance of developing sampling and testing protocols that optimize the probability of detecting PPV at the tree and block scales, because trees and blocks are what are targeted for eradication. There are at least two potential approaches that could be used to improve PPV detection efficiency at the tree scale. One is to increase the number of leaves sampled per tree; however, as sample size increases, virus titer from a single PPV-infected leaf becomes diluted, possibly below the ELISA detection threshold. Consequently, the increased probability of including a single PPVinfected leaf in a bulk sample is offset by dilution of virus titer, potentially increasing the number of false negatives. Group testing of multiple bulk samples would likely increase detection efficiency in both PPV eradication programs. For example, based on our simulations, detection efficiency would likely increase if ELISA tests were conducted on two 10-leaf bulk samples instead of a single 20-leaf bulk sample. Thus, detection efficiency could be increased in Ontario without increasing sampling costs to collect 20 leaves, although testing costs and labor would increase.

\section{Acknowledgments}

Funding for this project was provided by the USDA-ERS. We thank E. Wierenga, V. Galvin, and Canadian Food Inspection Agency personnel who collected the Prunus leaf samples for this study; and two anonymous reviewers who provided constructive comments on an earlier draft of this manuscript.

\section{Literature Cited}

Cambra, M., Asensio, M., Gorris, M. T., Perez, E., Camarasa, E., Garcia, J. A., Moya, J. J., Lopez-Abella, D., Vela, C., and Sanz, A. 1994. Detection of Plum pox potyvirus using monoclonal antibodies to structural and nonstructural proteins. EPPO Bull. 24:569-577.

Cambra, M., Capote, N., Myrta, A., and Llacer, G. 2006. Plum pox virus and the estimated costs associated with Sharka disease. EPPO Bull. 36:202-204.

Canadian Food Inspection Agency. 2011. Plum Pox Virus Monitoring and Management Program. Online publication. http://www.inspection.gc.ca/ plants/plant-protection/diseases/plum-pox-virus/monitoring-and managementprogram/eng/1323887724804/1323889930176

Capote, N., Bertolini, E., Olmos, A., Vidal, E., Martínez, M. C., and Cambra, M. 2009. Direct sample preparation methods for the detection of Plum pox virus by real-time RT-PCR. Int. Microbiol. 12:1-6.

Cohen, J. 1968. Weighted kappa: Nominal scale agreement provision for scaled disagreement or partial credit. Psychol. Bull. 70:213-220.

Gougherty, A. V., and Nutter, F. W., Jr. 2015. Impact of eradication programs on the temporal and spatial dynamics of Plum pox virus in Pennsylvania and Ontario Canada. Plant Dis. 99:593-603.
Hughes, G., Gottwald, T., and Levy, M. 2002. The use of hierarchical sampling in the surveillance program for Plum pox virus incidence in the United States. Plant Dis. 86:259-263.

Levy, L., Damsteegt, V., and Welliver, R. 2000. First report of Plum pox virus (Sharka disease) in Prunus persica in the United States. Plant Dis. 84:202.

Madden, L. V., Hughes, G., and van den Bosch, F. 2007. The Study of Plant Disease Epidemics. American Phytopathological Society, St. Paul, MN.

McNemar, Q. 1947. Note on the sampling error of the difference between correlated proportions or percentages. Pychometrika 12:153-157.

Mumford, R. A. 2006. Control and monitoring: Control of Plum pox virus in the United Kingdom. EPPO Bull. 36:315-318.

Myrta, A., Di Terlizzi, B., Savino, V., and Martelli, G. P. 2006. Control and monitoring: Monitoring and eradication of Sharka in south-east Italy over 15 years. EPPO Bull. 36:309-311.

NAPPO. 2004. Guidelines for phytosanitary action following detection of Plum pox virus. NAPPO Regional Standards for Phytosanitary Measures. Online publication. http://www.nappo.org/en/data/files/download/PDF/RSPM18-18-1004-e.pdf

NAPPO. 2013. Plum pox virus regulated areas removed in Orleans and Wayne counties, New York. Phytosanitary Alert System. Online publication. http:// www.pestalert.org/oprDetail.cfm?oprID $=557$

Nutter, F. W., Jr. 2007. The role of plant disease epidemiology in developing successful integrated disease management programs. Pages 43-77 in: General Concepts in Integrated Pest and Disease Management. Ciancio, A., and Mukerji, K. G., eds. Springer-Verlag, Dordrecht, The Netherlands.

Nutter, F. W., Jr., Byamukama, E., Coelho-Netto, R. A., Eggenberger, S. K., Gleason, M. L., Gougherty, A., Robertson, A. E., and Van Rij, N. 2011. Integrating GPS, GIS, and remote sensing technologies with disease management principles to improve plant health. Pages 59-90 in: GIS Applications in Agriculture-Invasive Species. S. Clay, ed. Taylor \& Francis Group LLC, Boca Raton, FL.

Ramel, M. E., Gugerli, P., and Bunter, M. 2006. Control and monitoring: Eradication of Plum pox virus in Switzerland. EPPO Bull. 36:312-314.

Ravelonandro, M., Vrient, A., Briard, P., and Mason, M. 2008. Controversial considerations between nucleotide diversity of the $3^{\prime}$ terminal region of Plum pox virus and differences in viral serotypes. J. Plant Pathol. 90(Suppl.):S1.47-S1.56.

Redding, R. C. 2009. Revision of quarantine and program orders; relating to Plum pox virus. Pa. Bull. Vol. 39, No. 46. Pa. Dep. Agric. Harrisburg.

Roberts, F., and Tesman, B. 2011. Applied Combinatorics, 2nd ed. CRC Press, Boca Raton, FL.

Snover-Clift, K. L., Clement, P. A., Jablonski, R., Mungari, R. J., Mavrodieva, V. A., Negi, S., and Levy, L. 2007. First report of Plum pox virus on plum in New York State. Plant Dis. 91:1512.

Speich, P. 2006. Control and monitoring: Plum pox virus quarantine situation in France. EPPO Bull. 36:307-308.

Stobbs, L. 2009. Plum pox virus: Studies on virus distribution and sampling methods. In: United States-Canada Plum pox virus Update Conf. Niagara Falls, Ontario, Canada.

Thompson, D. 2003. Woody host range of Canadian PPV-D. Page 7 in: NE1006 Eradication, Containment and/or Management of Plum Pox Disease (Sharka) Annu. Meet. NE1006, Shepardstown, WV. Online publication. http://www. lgu.umd.edu/lgu_v2/pages/reportMeet/62_min.pdf

Thompson, D. 2006. Control and monitoring: Control strategies for Plum pox virus in Canada. EPPO Bull. 36:302-304.

Thompson, D., McCann, M., MacLeod, M., Lye, D., Green, M., and James, D. 2001. First report of Plum pox potyvirus in Ontario, Canada. Plant Dis. 85:97.

USDA. 2007. Recovery plan for Plum pox virus (Sharka) of stone fruit. National Plant Disease Recovery System. Online publication. http://www.ars.usda.gov/ SP2UserFiles/Place/00000000/opmp/Plum\%20Pox\%20PPV\%2070222.pdf

USDA. 2013. DAS ELISA for detection of Plum pox virus using Agdia PPV ELISA kit. Online publication. http://www.aphis.usda.gov/plant_health/ plant_pest_info/plum_pox/downloads/ELISA\%20PPV.pdf

Vrient, A. 2006. External validation of Agdia's Plum pox virus (PPV) reagent set, catalog number SRA 31505. In: Rep. U. S. Dep. Agric. PPV Tech. Working Group, Elkhart, IN

Wang, A., Sanfacon, H., Stobbs, L., James, D., Thompson, D., Svircev, A., and Brown, D. 2006. Plum pox virus in Canada: Progress in research and future prospects for disease control. Can. J. Plant Pathol. 28:182-196.

Zadoks, J. C., and Schein, R. D. 1979. Epidemiology and Plant Disease Management. Oxford University Press, New York.

Zandarski, J., and Zych, A. 2005. A long-term strategy of sharka eradication in Poland. Phytopathol. Polonica 36:137-141 